Marquette University

e-Publications@Marquette

\title{
Feasibility, Acceptability, and Preliminary Outcomes of a Culturally Adapted Evidence-Based Treatment for Latino Youth With ADHD
}

\author{
Alyson C. Gerdes \\ Marquette University, alyson.gerdes@marquette.edu \\ Theresa Lauer Kapke \\ Marquette University \\ Margaret Grace \\ Marquette University \\ Al Castro \\ United Community Center
}

Follow this and additional works at: https://epublications.marquette.edu/psych_fac

Part of the Psychology Commons

\section{Recommended Citation}

Gerdes, Alyson C.; Kapke, Theresa Lauer; Grace, Margaret; and Castro, Al, "Feasibility, Acceptability, and Preliminary Outcomes of a Culturally Adapted Evidence-Based Treatment for Latino Youth With ADHD" (2021). Psychology Faculty Research and Publications. 426.

https://epublications.marquette.edu/psych_fac/426 
Marquette University

e-Publications@Marquette

\section{Psychology Faculty Research and Publications/College of Arts and Sciences}

This paper is NOT THE PUBLISHED VERSION; but the author's final, peer-reviewed manuscript. The published version may be accessed by following the link in the citation below.

Journal of Attention Disorders, (2019): 1-16. DOI. This article is (C SAGE publications and permission has been granted for this version to appear in e-Publications@Marquette. SAGE publications does not grant permission for this article to be further copied/distributed or hosted elsewhere without the express permission from SAGE publications.

\section{Feasibility, Acceptability, and Preliminary Outcomes of a Culturally Adapted Evidence- Based Treatment for Latino Youth With ADHD}

Ayson C. Gerdes

Marquette University, Milwaukee, WI, USA

Theresa L. Kapke

Marquette University, Milwaukee, WI, USA

Margaret Grace

Marquette University, Milwaukee, WI, USA

Al Castro

United Community Center, Milwaukee, WI, USA 


\section{Abstract}

Objective: To advance our knowledge about the most effective way to treat Latino youth with ADHD, the current feasibility and pilot study compared a culturally adapted evidence-based treatment (CAT) for ADHD to standard evidence-based treatment (EBT).

Method: Following a comprehensive ADHD assessment, 61 Latino families of school-aged children (mean age of 8 years) were randomly assigned to either CAT or standard EBT (i.e., parent management training).

Results: CAT outperformed standard EBT when examining homework completion and mother-reported treatment satisfaction. Apart from two trends favoring CAT, CAT and EBT both resulted in significant improvements in parent- and teacher-reported ADHD symptoms and functional impairment, as well as motherand father-reported parental functioning.

Conclusion: CAT outperformed standard EBT when examining several engagement and acceptability outcomes. CAT and EBT were equally effective when examining traditional treatment outcomes, which is impressive considering the robustness of standard EBT, especially when delivered by culturally competent staff.

\section{Keywords}

ADHD, parent management training, cultural adaptations, Latino, mental health disparities

\section{Introduction}

The last decade has seen a surge in the Latino population in the United States, an increased recognition of mental health disparities, and a call for more culturally sensitive treatment (Alegría, Vallas, \& Pumariega, 2010; Cabassa, Zayas, \& Hansen, 2006; Centers for Disease Control and Prevention [CDC], 2018; Sue, Zane, Nagayama Hall, \& Berger, 2009). There is a crucial need for research on effective treatments for Latino youth with ADHD, as it is one of the most common mental health disorders in childhood (American Psychiatric Association, 2013). Current evidence indicates that Latino parents perceive some aspects of parent management training (PMT), the leading psychosocial treatment for ADHD, negatively, which may diminish their engagement and motivation in the treatment process (Gerdes, Kapke, Lawton, Grace, \& Dieguez Hurtado, 2015). Thus, the current feasibility and pilot study compared a culturally adapted evidence-based treatment (CAT) for ADHD to standard evidence-based treatment (EBT) to advance our knowledge about the most effective way to treat Latino youth with ADHD.

\section{EBTs for Ethnic Minority Youth}

Recent estimates suggest that nearly one million Latino youth in the United States have ADHD, and the lifetime prevalence of ADHD in Latinos increased by 53\% from 2003 to 2007 (CDC, 2013; U.S. Census Bureau, 2015; Visser, Bitsko, Danielson, Perou, \& Blumberg, 2010). Despite these findings, Latino youth have not been well represented in treatment outcome studies examining PMT for ADHD (Huey \& Polo, 2008; Miranda et al., 2005). Although the Multimodal Treatment Study of Children With ADHD (MTA) examined the effects of ethnicity on treatment outcomes in children with ADHD (Arnold et al., 2003), these findings are unlikely to generalize to Latino families less oriented to U.S. mainstream culture (e.g., families who report Spanish as their only or predominant language and who report higher orientation to Latino culture than U.S. mainstream culture) due to the inclusion criteria that was employed. Participation in the MTA required parents to be fluent English speakers and to agree to medication if their child was randomly assigned to that treatment condition, yet many Latino parents do not believe medication to be a suitable treatment for ADHD (Arcia, Fernández, \& Jáquez, 2004). Thus, to date, there is little empirical evidence to support the effectiveness of PMT for ADHD in Latino families, especially families less oriented to U.S. mainstream culture. 
Importantly, initial reviews examining EBT outcomes in ethnic minority youth have reported promising findings for many disorders, including conduct problems, mood disorders, and anxiety disorders; however, most studies summarized in these reviews compared ethnic minority youth to European American youth to determine whether groups differed regarding their response to standard EBT or examined ethnic-minority-only samples to determine the effects of a culturally adapted treatment (Huey \& Polo, 2008; Miranda et al., 2005). Baumann and colleagues (2015) conducted a recent review focused on evidence-based parent training programs and concluded that studies examining "rigorously" developed culturally adapted parent training programs are rare. Furthermore, although the studies summarized in the Baumann review typically demonstrated the superiority of culturally adapted interventions over control or wait list groups, only one examined whether a culturally adapted evidence-based program outperformed a standard evidence-based program.

In fact, except for an intervention examining youth with conduct problems (McCabe \& Yeh, 2009), no research has directly compared a culturally adapted intervention to a standard evidence-based intervention to determine whether an adapted treatment actually outperforms an existing EBT. Even more unique, McCabe, Yeh, Lau, and Argote (2012) also conducted a follow-up of their direct comparison. They demonstrated that both Parent-Child Interaction Therapy (PCIT) and its cultural adaptation (Guiando a Niños Activos [GANA]) maintained the posttreatment improvements documented in the original study and that GANA, but not PCIT, outperformed treatment as usual at follow-up. Treatment outcome studies of this nature are imperative, as they will allow clinicians and researchers to determine whether it is necessary to adapt EBTs for use with ethnic minority families.

\section{Latino Youth in the United States}

Latinos are the largest, most rapidly growing ethnic minority group in the United States, with estimates predicting that nearly $40 \%$ of the children in the country will identify as Latino by 2060 (U.S. Census Bureau, 2015). Recent studies suggest that although Latino and European American youth exhibit similar rates of ADHD symptomatology, Latino youth are less likely to be diagnosed and receive treatment (Eiraldi \& Diaz, 2010; Leslie, Lambros, Aarons, Haine, \& Hough, 2008; Morgan, Hillemeier, Farkas, \& Maczuga, 2014). Many barriers contributing to these mental health disparities have been identified. Stigma associated with seeking mental health services, poverty, lack of health insurance, transportation and scheduling difficulties, and language differences, as well as negative interactions with mental health providers and use of culturally insensitive assessment tools, have been reported (Alegría \& Woo, 2009; Gerdes, Lawton, Haack, \& Dieguez Hurtado, 2013; Kouyoumdjian, Zamboanga, \& Hansen, 2003).

\section{ADHD Treatment}

ADHD is one of the most commonly diagnosed mental health disorders in youth. To receive a diagnosis of ADHD, both developmentally inappropriate levels of inattention and/or hyperactivity/impulsivity and functional impairment must be present (American Psychiatric Association, 2013). Follow-up research of youth diagnosed with ADHD suggests that these children continue to experience impairment in various domains as they move into adolescence and adulthood (Biederman et al., 2012). Research supports the effectiveness of PMT at improving child behavior and parental functioning in youth with ADHD from predominantly European American families, as well as Latino families more oriented to U.S. mainstream culture (Arnold et al., 2003; Gerdes, Haack, \& Schneider, 2012; Pelham \& Fabiano, 2008); however, no published research has examined its effectiveness with Latino families less oriented to U.S. mainstream culture.

The increased recognition and need for culturally adapted treatments have resulted in several researchers putting forth guidelines and models for how to adapt existing EBTs most effectively (Bernal \& Domenech Rodríguez, 2012; Bernal, Jiménez-Chafey, Domenech, \& Rodríguez, 2009). Employing many of these guidelines 
and following the Ecological Validity Model (Baumann et al., 2015), with a particular focus on language, content, goals, and persons, Gerdes and colleagues' (2015) cultural adaptation of PMT for Latino families less oriented to U.S. mainstream culture supports the need for cultural adaptations that will ensure parental engagement and motivation in treatment. Specifically, focus groups with Latino parents suggested that several sessions of standard PMT (i.e., time out and token economy) likely would not be well received by families and that several sessions (e.g., positive reinforcement) should include more culturally congruent rationales for skills, as the strategies are inconsistent with common parenting beliefs held by some Latino parents (e.g., some Latino parents may be less likely to provide praise, especially for expected behavior, than their European American counterparts; for more detail, see Gerdes et al., 2015). Integrating this focus group data with research examining Latino cultural values and recommendations from bicultural mental health providers, Gerdes and colleagues $\underline{2015)}$ developed a culturally adapted PMT program for Latino youth with ADHD. Treatment outcomes from an initial pilot study were positive, although the sample size was small $(n=5)$, and no comparison to standard EBT was examined (Gerdes et al., 2015).

\section{Current Study}

Thus, the current larger feasibility and pilot study aimed to close the existing gap in our knowledge about effective psychosocial treatments for Latino families by determining whether CAT for ADHD outperforms standard EBT when examining engagement and acceptability outcomes, as well as symptomatology and child and parental functioning. The current study is the first to compare a culturally adapted treatment for ADHD to standard EBT, and it is the first to examine treatment outcomes for ADHD in school-aged Latino youth from families less oriented to U.S. mainstream culture (i.e., families living in the United States for more than a decade who report Spanish as their only or predominant language and who report higher orientation to Latino culture than U.S. mainstream culture on measures of acculturation). Given the pilot nature of the study, as well as the fact that the vast majority of treatment outcome studies examining cultural adaptations, to date, have not employed a direct comparison of a culturally adapted treatment to an EBT, specific predictions were not made; however, three aims guided the analyses. Aim 1 was to explore whether CAT results in better engagement and acceptability outcomes (i.e., parental attendance, retention, engagement, and satisfaction) than standard EBT. Aim 2 was to explore whether CAT results in improvements in ADHD symptomatology and functioning and to explore whether the CAT results in similar or greater improvements than standard EBT. Aim 3 was to explore whether CAT results in improvements in parental and family functioning (i.e., parenting stress, parental efficacy, and family chaos) and to explore whether CAT results in similar or greater improvements than standard EBT.

\section{Method}

\section{Participants}

Families were recruited in a moderate-sized city in the Midwest through local schools, a community mental health clinic serving the Latino community, and word of mouth referrals. To be eligible to participate, children had to self-identify as Latino, be between the ages of 5 and 13 years at the time of the initial assessment, receive a primary diagnosis of $A D H D$, and be on a stable dose of medication for at least 2 weeks prior to the assessment if they were being medicated for ADHD. Children were excluded if parents or teachers reported behaviors suggesting the presence of intellectual disability (ID), autism spectrum disorder (ASD), or a psychotic disorder or if clinicians noted these behaviors during the assessment, in which case families were provided with appropriate resources. Participating parents had to self-identify as Latino, be fluent in Spanish, be able and willing to provide informed consent and comply with the study procedures, including being assigned to one of two treatments, and have no immediate plans to pursue other treatment for their child's ADHD or to change their child's ADHD medication (if already medicated) over the next 8 weeks.

Over the course of 2 years, 74 families were recruited and assessed. Sixty-one of these families were randomly assigned to a treatment condition; 10 did not meet criteria for ADHD, one met exclusion criteria (i.e., active 
psychosis), and two did not finish the assessment (see Figure 1). Of the children whose families were assigned to a treatment condition, $100 \%$ identified as Latino, and $72 \%$ were male with a mean age of 7.98 years $(S D=2.57$; range $=5-13$ years). Twenty-six received a diagnosis of ADHD, Predominantly Inattentive Presentation, seven received a diagnosis of ADHD, Predominantly Hyperactive-Impulsive Presentation, and 28 received a diagnosis of ADHD, Combined Presentation. Twelve of these children were on ADHD medication. Eighteen children had a comorbid diagnosis (14 with a conduct-related disorder and four with a mood/anxiety disorder). The mean socioeconomic status (SES) of families suggests that the average family would fall into the semiskilled worker category (Hollingshead, 1975). Eighty-two percent of mothers and 85\% of fathers identified Mexico as their country of origin; $68 \%$ of mothers and $81 \%$ of fathers reported living in the United States for more than 10 years; and $75 \%$ of mothers and $85 \%$ of fathers identified Spanish as their only or predominant language. Means on acculturation measures suggests that mothers and fathers were more oriented to Latino culture than U.S. mainstream culture. Demographic and cultural variables broken down by treatment condition may be found in Table 1.

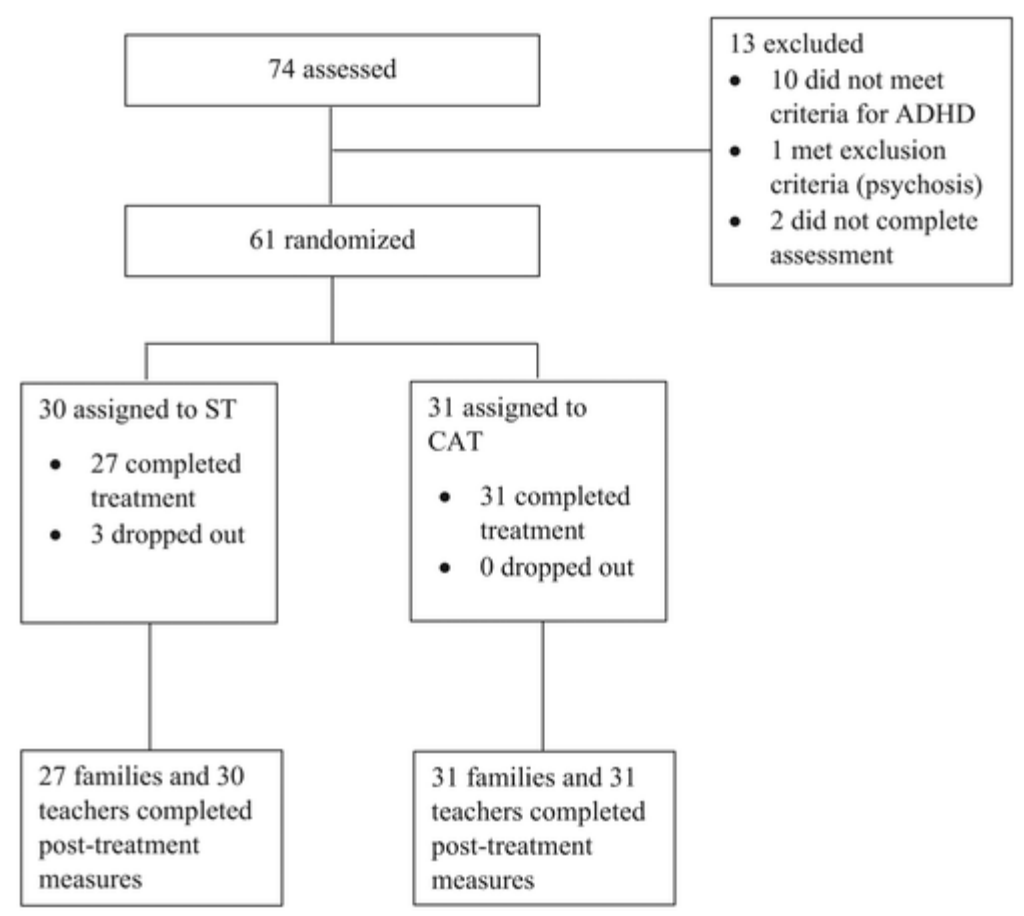

Figure 1. CONSORT figure.

Note. ST = standard evidence-based treatment; CAT = culturally adapted evidence-based treatment.

Table 1. Parent and Child Demographic and Cultural Variables.

\begin{tabular}{|l|l|l|l|}
\hline & CAT & ST & Statistic \\
\hline Child variables & & & \\
\hline Age, $M(S D)$ & $7.84(2.49)$ & $8.13(2.68)$ & $F(1,59)=0.20$ \\
\hline Gender, $n(\%)$ & & & $\chi^{2}(1)=0.13$ \\
\hline Female & $8(25.81)$ & $9(30.00)$ & \\
\hline Male & $23(74.19)$ & $21(70.00)$ & \\
\hline ADHD subtype, $n(\%)$ & & & $\chi^{2}(2)=2.08$ \\
\hline Hyperactive/impulsive & $3(9.68)$ & $4(13.33)$ & \\
\hline Inattentive & $16(51.61)$ & $10(33.33)$ & \\
\hline Combined & $12(38.71)$ & $16(53.33)$ & \\
\hline
\end{tabular}




\begin{tabular}{|c|c|c|c|}
\hline Medication status, $n$ (\%) & & & $\chi^{2}(1)=0.004$ \\
\hline Yes & $6(19.35)$ & $6(20.00)$ & \\
\hline No & $25(80.65)$ & $24(80.00)$ & \\
\hline Comorbidity status, $n$ (\%) & & & $\chi^{2}(1)=0.42$ \\
\hline Yes & $8(25.81)$ & $10(33.33)$ & \\
\hline No & $23(74.19)$ & $20(66.67)$ & \\
\hline Referral source, $n(\%)$ & & & $\chi^{2}(2)=0.06$ \\
\hline Medical institution & $7(22.58)$ & $6(20.00)$ & \\
\hline School & $13(41.94)$ & $13(43.33)$ & \\
\hline Self & $11(35.48)$ & $11(36.67)$ & \\
\hline \multicolumn{4}{|l|}{ Parent variables } \\
\hline Marital status, $n(\%)$ & & & $\chi^{2}(1)=0.84$ \\
\hline Married/cohabitating & $23(74.19)$ & $19(63.33)$ & \\
\hline Separated/divorced & $8(25.81)$ & $11(36.67)$ & \\
\hline Family SES, $M(S D)$ & $23.47(11.34)$ & $23.38(11.10)$ & $F(1,59)=0.001$ \\
\hline Mother's age $M(S D)$ & $34.27(4.86)$ & $36.40(4.93)$ & $F(1,58)=2.85$ \\
\hline Father's age $M(S D)$ & $37.94(7.12)$ & $40.70(12.85)$ & $F(1,25)=0.52$ \\
\hline Mother's country of origin, $n(\%)$ & & & $\chi^{2}(2)=1.18$ \\
\hline Mexico & $23(76.67)$ & $26(86.67)$ & \\
\hline Puerto Rico & $1(3.33)$ & $1(3.33)$ & \\
\hline Other & $6(20.00)$ & $3(10.00)$ & \\
\hline Father's country of origin, $n(\%)$ & & & $\chi^{2}(2)=0.72$ \\
\hline Mexico & $14(82.35)$ & $9(90.00)$ & \\
\hline Puerto Rico & $1(6.25)$ & $0(0.00)$ & \\
\hline Other & $2(12.50)$ & $1(10.00)$ & \\
\hline \multicolumn{4}{|l|}{ Mother acculturation, $M(S D)$} \\
\hline MOS/LOS & $4.41(0.48)$ & $4.51(0.42)$ & $F(1,58)=0.82$ \\
\hline LAV & $3.94(0.37)$ & $3.93(0.53)$ & $F(1,58)=0.02$ \\
\hline AOS & $2.52(0.84)$ & $2.36(0.90)$ & $F(1,58)=0.50$ \\
\hline MV & $2.78(0.50)$ & $2.83(0.60)$ & $F(1,58)=0.12$ \\
\hline \multicolumn{4}{|l|}{ Father acculturation, $M(S D)$} \\
\hline MOS/LOS & $4.12(0.61)$ & $4.22(0.53)$ & $F(1,24)=0.20$ \\
\hline LAV & $4.04(0.43)$ & $3.99(0.47)$ & $F(1,24)=0.06$ \\
\hline AOS & $2.67(0.65)$ & $2.27(1.01)$ & $F(1,24)=1.55$ \\
\hline MV & $3.03(0.41)$ & $2.83(0.67)$ & $F(1,24)=0.92$ \\
\hline
\end{tabular}

Note. Hollingshead's Four Factor Index of Social Status (Hollingshead, 1975) was used to compute SES for each family. Child $n=61$; Mother $n=60$; Father $n=27$. CAT = culturally adapted evidence-based treatment; $S T=$ standard evidence-based treatment; SES = socioeconomic status; MOS/LOS = ARSMA-II Mexican/Latino Orientation Scale; MACVS LAV = Latino American Values Scale; ARSMA-II AOS = Anglo Orientation Scale; MACVS MV = Mainstream Values Scale.

\section{Procedure}

The current study was approved by the Institutional Review Board at Marquette University. Written informed consent was obtained from parents and teachers, and written assent was obtained from children. Parents and teachers were compensated with gift cards for completing assessment and posttreatment measures. Assessment measures were completed prior to the start of treatment, and posttreatment measures were completed during the last week of treatment. 


\section{Assessment and Diagnosis}

Each child received a multi-method, multi-informant assessment, including parent, teacher, and child interviews and measures. Parents participated in a semi-structured clinical interview focused on the presenting problem and psychosocial history in Spanish, as well as the Spanish translation of the Disruptive Behavior Disorders (DBD) Structured Parent Interview, a diagnostic interview aimed at diagnosing ADHD and common comorbid disorders (Gerdes et al., 2015; Pelham, 2002). Teachers participated in a brief, in-person teacher interview focused on the presenting problem. All assessments were completed by one of two bilingual clinical psychology doctoral students.

Parents and teachers also completed several measures, including the Child Behavior Checklist (CBCL)/Teacher's Report Form (TRF; Achenbach \& Rescorla, 2001), Parent/Teacher DBD Rating Scale (Pelham, Gnagy, Greenslade, \& Milich, 1992), and ADHD-FX Scale (Haack, Gerdes, Lawton, \& Schneider, 2016). If a child was medicated for ADHD, unmedicated ratings on the DBD Rating Scale and ADHD-FX Scale were used for making diagnosis decisions. Finally, children participated in an unstructured clinical interview and completed the Children's Depression Inventory 2 (CDI 2) and Revised Children's Manifest Anxiety Scale-Second Edition (RCMAS-2; Kovacs \& MHS Staff, 2011; Reynolds \& Richmond, 2008). All measures are described in more detail below.

Consistent with recommendations from the American Academy of Pediatrics and previous research (Lahey et al., 2004), diagnostic decisions, including subtype classifications, were based on all sources of information gathered during the assessment, including clinical interviews, observations, and measures to determine whether Diagnostic and Statistical Manual of Mental Disorders (5th ed.; DSM-5; American Psychiatric Association, 2013) criteria for ADHD were met. Specifically, clinical doctoral-level students and a licensed supervisor and ADHD expert made a final clinical judgment on severity of each DSM symptom for every child assessed. If it was determined that the child met criteria for ADHD and all other study criteria were met, the family was randomly assigned to one of two treatment conditions described below. Families and teachers were blind to treatment condition. Regardless of treatment condition, parent groups consisted of seven to eight families, resulting in four groups for each treatment condition.

\section{Treatment}

\section{Standard EBT}

Thirty families were assigned to standard EBT. Standard EBT consisted of eight group PMT sessions and an individualized behavioral classroom intervention in the form of a Daily Report Card (DRC). Consistent with standard outpatient therapy, sessions were held weekly in the evening at a university-based ADHD Clinic for

approximately $2 \mathrm{hr}$. Childcare and snacks were provided. Sessions were based on Barkley's (1997) manual and included DRC implementation, effective instructions, time out, positive reinforcement, token economy, planning ahead strategies, takeover of the DRC, and closing/wrap-up. All PMT groups were coled in Spanish by a bilingual clinical psychology doctoral student and a bilingual, bicultural social worker, both of whom had training in childhood ADHD and cultural competence (described in more detail below). Integrity checklists were completed by well-trained, bilingual undergraduate students each week (also described in more detail below).

\section{CAT}

Thirty-one families were assigned to CAT (Gerdes et al., 2015). CAT also consisted of eight group parent training classes and an individualized DRC; however, all classes were adapted and/or newly developed to be more culturally appropriate, and two home visits were added. Classes were held weekly in the evening at a neighborhood community center serving Latino families for approximately $2 \mathrm{hr}$. Childcare and dinner from a local Mexican restaurant were provided. Classes included DRC implementation, effective instructions, consistent consequences, positive attention and ignoring, managing routines-homework, managing routines-checklists, takeover of the DRC, and final tips for success. All CAT groups were coled in Spanish by a different bilingual 
clinical psychology doctoral student and the same bilingual, bicultural social worker who coled the PMT groups; both received training in childhood ADHD and cultural competence. Integrity checklists were completed by welltrained, bilingual undergraduate students each week.

Briefly, all classes were adapted to include additional in-session role-plays and parental coaching, video demonstrations of skills, more visually appealing handouts, frequent discussion and review of child's progress toward home- and school-based goals with the use of progress graphs, and efforts to include extended family members in treatment. Class-specific adaptations included the addition of relevant cultural reminders; culturally congruent rationales for skills and terminology (e.g., the rationale for the DRC is to empower parents and improve family communication); open discussion of parental concerns about skills and expectations for treatment (e.g., time is set aside to discuss concerns about rewarding expected behavior and about taking away privileges rather than spanking for misbehavior); emphasis of culturally valued, positive outcomes for the family with consistent use of the skills; and time dedicated to establishing a good working relationship between the parent and teacher. Finally, two classes were completely replaced with newly developed, more culturally congruent classes (i.e., consistent consequences replaced time out and managing routines replaced token economy) with home visits following each of these classes (see Gerdes et al., 2015, for more detail).

\section{Clinician Training and Integrity Checklists}

\section{Clinician training}

The bilingual clinical psychology doctoral students who served as the primary leads for the two treatment conditions received training regarding evidence-based assessment and treatment of childhood ADHD from the first author as part of their clinical training within their doctoral program. Coleaders for both treatments (i.e., the clinical psychology doctoral students and the bilingual, bicultural social worker) participated in an intensive training program specific to the research protocol over the course of 4 weeks, also led by the first author. The program included psychoeducation about childhood ADHD and thorough review of both treatment manuals, including a point-by-point review of each session/class outline. The relevant integrity checklist also was reviewed with each session/class outline to ensure adherence to both treatment manuals. Coleaders also participated in a 2-day cultural competence workshop provided by an expert in culturally competent mental health treatment and culturally adapted parent training programs. Throughout treatment, coleaders participated in weekly group supervision with the first author; adherence to integrity checklists also was discussed each week.

\section{Integrity checklists}

The bilingual undergraduate students who completed the integrity checklists participated in the same intensive training program, cultural competence workshop, and weekly supervision described above. To ensure adherence to the treatment manuals, these students were trained to check off each bullet point in the session/class outline as it was discussed and to alert the coleaders as soon as was feasible if a bullet point was missed. Given that the students were present during the sessions/classes, this system allowed the team to achieve $100 \%$ adherence to both treatment manuals.

\section{Assessment Only Measures}

\section{Client information form}

A client information form was completed by the primary caregiver as part of the comprehensive assessment. The form was used to collect basic demographic information, such as child age, gender, and ethnicity, as well as parental education and occupation. 


\section{$\mathrm{CBCL}$ and TRF}

The age-appropriate version of the CBCL and TRF (Achenbach \& Rescorla, 2001), parent- and teacher-report measures, respectively, assessing a broad range of child behaviors, was completed by the primary caregiver and teacher as part of the assessment. The version for 6 - to 18 -year-olds consists of 112 to 113 items, and the version for $1 \frac{1}{2}$ - to 5 -year-olds consists of 100 items. Sound psychometrics for the Spanish translation of the $\mathrm{CBCL}$ and English version of the TRF completed in the current study have been reported (Achenbach \& Rescorla, 2001; Rubio-Stipec, Bird, Canino, \& Gould, 1990).

\section{2 and RCMAS-2}

The CDI 2 and RCMAS-2 (Kovacs \& MHS Staff, 2011; Reynolds \& Richmond, 2008), self-report measures of depressive and anxiety symptoms, respectively, were completed by children who met the minimum age requirement at the time of the assessment. The CDI 2 consists of 28 items on a 3-point scale; the RCMAS-2 consists of 49 items presented in a yes or no format. Children completed the measures in either English or Spanish, depending on their language preference. Both English and Spanish versions of both measures have

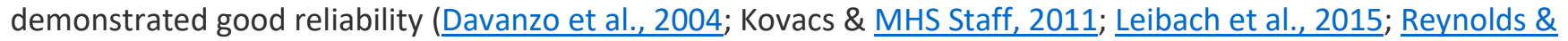
Richmond, 2008).

\section{Economic and Cultural Measures}

\section{SES}

Hollingshead's Four Factor Index of Social Status (Hollingshead, 1975) was used to compute SES for each family. Specifically, education and occupation of the parents currently living in the home at the time of the assessment were used to compute family SES. This information was provided in the Client Information Form described above.

\section{Acculturation Rating Scale for Mexican Americans-II (ARSMA-II)}

The ARSMA-II, a self-report measure of behavioral acculturation, was completed individually by mothers and fathers prior to treatment (Cuellar, Arnold, \& Maldonado, 1995). It consists of 30 items, making up two subscales-Anglo Orientation (AOS) and Mexican/Latino Orientation (MOS/LOS), which were examined in the current study. Each item is rated on a 5-point scale, ranging from "not at all" to "extremely often/almost always," with higher scores indicating greater orientation to Anglo or Mexican/Latino culture, depending on which subscale is being examined. Psychometric properties for the Spanish translation have been wellestablished (Cuellar et al., 1995) and were maintained when making minor wording changes to allow for a broader use of the measure (e.g., "Mexican" was changed to "Latino"; Steidel \& Contreras, 2003). In the current study, the measure displayed good reliability for the AOS and MOS/LOS subscales for mothers (Cronbach's $\alpha \mathrm{s}=$ .91 and .82 , respectively) and fathers (Cronbach's $\alpha$ s $=.92$ and .83 , respectively).

\section{Mexican American Cultural Values Scale for adolescents and adults (MACVS)}

The MACVS (Knight et al., 2010), a self-report measure of cultural value orientation, was completed individually by mothers and fathers prior to treatment. It consists of 50 items, making up two subscales-Mainstream Values (MV) and Latino American Values (LAV), both of which were examined in the current study. Items are rated on a 5-point scale, ranging from not at all to completely believe, with higher scores indicating greater orientation to MV or LAV, depending on which subscale is being examined. Strong psychometrics for the Spanish translation have been demonstrated (Knight et al., 2010). In the current study, the measure displayed good reliability for the MV and LAV for mothers (Cronbach's $\alpha s=.70$ and .88 , respectively) and fathers (Cronbach's $\alpha s=.73$ and .89 , respectively). 


\section{Child Symptomatology and Functional Impairment Measures}

\section{DBD Rating Scale}

The DBD Rating Scale, a parent- and teacher-report measure of the DSM symptoms of ADHD, Oppositional Defiant Disorder, and Conduct Disorder, was completed by the primary caregiver and primary teacher both preand posttreatment (Gerdes et al., 2013; Pelham et al., 1992). It consists of 45 items that are rated on a 4-point scale, ranging from not at all present to very much present. For purposes of the current study, a mean was computed for the nine inattentive items and the nine hyperactive/impulsive items, with higher scores representing greater symptomatology in those domains. Research demonstrates sound psychometrics for the original English version (completed by teachers; Pelham et al., 1992) and the Spanish translation (completed by parents; Gerdes et al., 2013). In the current study, the measure displayed good reliability for parent reports of inattention and hyperactivity/impulsivity (Cronbach's $\alpha s=.90$ and .89 , respectively) and teacher reports of inattention and hyperactivity/impulsivity (Cronbach's $\alpha \mathrm{s}=.91$ and .88 , respectively).

\section{ADHD-FX Scale}

The ADHD-FX Scale (Haack, Gerdes, et al., 2016), a parent- and teacher-report measure of functional impairment commonly experienced by youth with ADHD, was completed by the primary caregiver and primary teacher both pre- and posttreatment. The parent portion consists of 32 items across the domains of home and school, and the teacher portion consists of 19 items in the school setting. Items are rated on a 4-point scale, ranging from not at all to a lot. For purposes of the current study, a mean was computed for parent-report of functional impairment in the home and for teacher-report of functional impairment at school, with higher scores representing greater impairment. It is normed for youth aged 5 to 15 years and demonstrates sound psychometrics for both the English version (completed by teachers) and Spanish version (completed by parents; Haack, Gonring, Harris, Gerdes, \& Pfiffner, 2016). In the current study, the measure displayed good reliability for parent-reported home impairment (Cronbach's $\alpha=.95$ ) and teacher-reported school impairment (Cronbach's $\alpha=.94)$.

\section{Parental and Family Functioning Measures}

Parenting Stress Index-4-Short Form (PSI-4-SF)

The PSI-4-SF (Abidin, 2012), a parent-report measure of parenting stress, was completed individually by mothers and fathers both pre- and posttreatment. It consists of 36 items rated on a 5-point scale, ranging from strongly agree to strongly disagree. Total parenting stress (sum of all 36 items) reported by each parent who participated in treatment was used in the current study, with higher scores indicating greater levels of parenting stress. Kim, Lau, and Chorpita (2016) reported sound psychometrics for the Spanish translation. Good internal consistency was found in the current study (.92 for mothers and .93 for fathers).

\section{Parenting Sense of Competence Scale (PSOC)}

The Parenting Efficacy subscale of the PSOC (Johnston \& Mash, 1989), a parent-report measure of parental efficacy, was completed individually by mothers and fathers both pre- and posttreatment. Parents rated seven items on a 6-point scale, ranging from strongly agree to strongly disagree. For purposes of the current study, a mean was computed for each parent who participated in treatment, with higher scores suggesting higher perceived parental efficacy. Good internal consistency and validity have been reported for the Spanish translation used in the current study (Haack, Gerdes, Schneider, \& Dieguez Hurtado, 2011). Good internal consistency was found in the current study (.81 for mothers and .79 for fathers).

Confusion, Hubbub, and Order Scale (CHAOS)

The CHAOS (Matheny, Wachs, Ludwig, \& Phillips, 1995), a parent-report measure of environmental chaos in the home, was completed individually by mothers and fathers both pre- and posttreatment. Parents rated 15 items on a 6-point scale, ranging from strongly agree to strongly disagree. For purposes of the current study, a sum 
was computed for each participating parent, with higher scores indicating more reported chaos in the home. Haack and colleagues (2011) reported good reliability and validity for the Spanish translation used in the current study. Good internal consistency was found in the current study (.77 for mothers and .81 for fathers).

\section{Additional Treatment Outcome Measures}

\section{Treatment attendance and retention}

The number of sessions/classes attended was recorded for each family. Attendance was defined as the number of sessions/classes attended by at least one parent. Retention was operationalized as family completion of the final scheduled session/class.

\section{Engagement}

Parents were assigned specific weekly homework related to practicing and/or implementing the parenting strategy taught during that week's group or monitoring behaviors outlined on the treatment plan and were asked to bring the completed homework sheet to session/class each week. Engagement was measured by examining how frequently parents completed these homework assignments, for which a percentage of completed homework assignments was computed for each family. Coleaders for both treatment groups also rated each participating parent's level of engagement on a 5-point scale $(1=$ very low to $5=$ very high $)$ at the end of treatment, and a mean of the coleaders' ratings was computed for each participating parent. If two parents participated, a mean of both parents' ratings was computed. These subjective ratings of parental engagement were based on the observed quality of parent participation and effort during the sessions/classes.

\section{Therapy Attitude Inventory (TAI)}

The TAI (Eyberg, 1993), a 10-item parent-report measure assessing parental satisfaction with treatment, was completed individually by mothers and fathers posttreatment. Parents rated each item on a 5-point scale; a total score was computed, with higher scores indicating greater satisfaction with treatment. Good internal consistency and validity have been demonstrated for the English version (Eisenstadt, Eyberg, McNeil, Newcomb, \& Funderburk, 1993). The TAl was translated to Spanish by the first author's research team. Good internal consistency was found in the current study (.84 for mothers and .81 for fathers).

Data analytic plan

All parent and child measures were completed and returned to research staff who were present during completion, and teacher measures were returned to research staff in person. Thus, missing data were almost nonexistent. If an item was unintentionally left blank, the measure was returned for completion. The data analytic plan for preliminary analyses consisted of analyses of variance (ANOVAs) and chi-square tests for independence to examine demographic and cultural differences between treatment conditions, and correlations, chi-square tests for independence, and independent-samples $t$ tests to examine demographic variables in relation to treatment outcome variables. The data analytic plan for primary analyses included independent-samples $t$ tests and one chi-square test for independence to examine engagement and acceptability outcomes, paired-samples $t$ tests to examine ADHD symptomatology, child functioning, and parent and family functioning outcomes by treatment condition, and repeated-measures ANOVAs to examine whether a treatment condition resulted in better treatment outcomes. SPSS 24 was employed to run all analyses. 


\section{Results}

\section{Preliminary Analyses}

\section{Demographic and cultural variables}

To examine possible demographic and/or cultural differences between treatment conditions, ANOVAs and chisquare tests for independence were examined. As Table 1 indicates, no significant differences between treatment conditions emerged for demographic variables (i.e., child age, child gender, ADHD subtype, medication status, comorbidity status, referral source, parents' marital status, family SES, mother/father age, and mother/father country of origin). Similarly, no significant differences emerged for cultural variables (i.e., mother- and father-reported behavioral and cognitive acculturation).

\section{Further examination of potential covariates}

Prior to conducting our primary analyses, correlations, chi-square tests for independence, and independentsamples $t$ tests were conducted for key demographic variables (i.e., child age, child gender, medication status, parents' marital status, and family SES) and all treatment outcome variables to determine whether any covariates needed to be included in our primary analyses. Results were nonsignificant, and no covariates were included in the primary analyses.

\section{Primary Analyses}

\section{Aim 1-Engagement and acceptability outcomes}

Independent-samples $t$ tests and one chi-square test for independence were employed to examine whether CAT resulted in better engagement and acceptability outcomes than standard EBT. Specifically, $t$ tests compared treatments on four outcomes-parental attendance, homework completion (objective measure of engagement), therapist-rated engagement, as well as mother- and father-reported satisfaction with treatment; a chi-square compared treatments on retention. As Table 2 indicates, CAT outperformed standard EBT when examining homework completion and mother-reported treatment satisfaction. Examination of the means indicated that families who participated in CAT demonstrated greater engagement by completing their homework more frequently than families who participated in standard EBT, and mothers reported greater satisfaction with CAT than standard EBT. The effect sizes were medium to large. Two additional marginally significant findings emerged for attendance and retention; parents who participated in CAT attended more treatment sessions/classes and were more likely to complete treatment than parents who participated in standard EBT. The effect sizes were small to medium.

Table 2. Results of Independent Samples t Tests and Chi-Square Test for Independence for Engagement and Acceptability Outcomes by Treatment Condition.

\begin{tabular}{|c|c|c|c|c|c|c|c|c|c|c|}
\hline & $\begin{array}{l}\text { Treatment } \\
\text { condition }\end{array}$ & & & & & & & & & \\
\hline & CAT & & & ST & & & & & & Cohen's \\
\hline Variables & $M$ & SD & $\mathbf{n}$ & M & SD & $\mathbf{n}$ & $95 \% \mathrm{Cl}$ & $\mathbf{t}$ & df & d \\
\hline Attendance & 7.52 & 0.93 & 31 & 6.93 & 1.46 & 30 & $\begin{array}{l}{[-1.21} \\
0.04]\end{array}$ & $-1.87^{\dagger}$ & 59 & 0.48 \\
\hline $\begin{array}{l}\text { HW } \\
\text { completion }\end{array}$ & 88.83 & 13.63 & 31 & 68.95 & 24.36 & 30 & $\begin{array}{l}{[-0.30,-} \\
0.10]\end{array}$ & $-\overline{3.92 * * *}$ & 45.22 & 1.00 \\
\hline $\begin{array}{l}\text { TR } \\
\text { engagement }\end{array}$ & 4.43 & 0.45 & 31 & 4.13 & 1.11 & 30 & $\begin{array}{l}{[-0.75} \\
0.14]\end{array}$ & -1.38 & 38.11 & 0.35 \\
\hline Mother TAI & 46.93 & 3.17 & 30 & 44.74 & 3.57 & 27 & $\begin{array}{l}{[-3.98,-} \\
0.40]\end{array}$ & $-2.46^{*}$ & 55 & 0.65 \\
\hline
\end{tabular}




\begin{tabular}{|l|l|l|l|l|l|l|l|l|l|l|}
\hline Father TAI & 46.76 & 3.25 & 17 & 44.89 & 2.89 & 9 & $\begin{array}{l}{[-4.54,} \\
0.79]\end{array}$ & -1.45 & 24 & 0.60 \\
\hline & CAT & $\mathbf{n}$ & $\mathbf{S T}$ & & & & & & \\
\hline & $\mathbf{n}$ & $\mathbf{n}$ & $\mathbf{\%}$ & $\mathbf{N - 1} \mathbf{2}$ & & $\mathbf{d f}$ & $\mathbf{\Phi}$ \\
\hline Retention No & 0 & 0.00 & 3 & 100.00 & $3.21+$ & & 1 & 0.23 \\
\hline Yes & 31 & 53.45 & 27 & 46.55 & & & & & \\
\hline
\end{tabular}

Aim 2-ADHD symptomatology and functioning outcomes

Paired-samples $t$ tests were employed to examine whether CAT resulted in improvements in ADHD symptomatology and functioning. Specifically, $t$ tests compared pre-post treatment scores on six outcomesparent- and teacher-reported inattention, parent- and teacher-reported hyperactivity/impulsivity, parentreported functioning at home, and teacher-reported functioning at school. As Table 3 indicates, significant findings emerged for parent- and teacher-reported inattention and parent- and teacher-reported functioning. A marginally significant finding also emerged for parent-reported hyperactivity/impulsivity. Examination of the means demonstrated that following CAT, parents and teachers reported fewer ADHD symptoms and less functional impairment than they did prior to treatment. The effect sizes for significant findings were medium, with several approaching large. For comparison, paired-samples $t$ tests for standard EBT also are presented in Table 3.

Table 3. Results of Paired Samples t Tests for ADHD Symptomatology and Functioning Outcomes at Pretreatment (Time 1) and Posttreatment (Time 2).

\begin{tabular}{|c|c|c|c|c|c|c|c|c|c|c|c|}
\hline & & & CAT & & & & & & & & \\
\hline & Time 1 & & & & Time 2 & & & & & & \\
\hline & $M$ & $S D$ & $n$ & & $M$ & $S D$ & $n$ & $95 \% \mathrm{Cl}$ & $t$ & $d f$ & Cohen's d \\
\hline P DBDin & 1.57 & 0.77 & 31 & & 1.13 & 0.56 & 31 & {$[0.21,0.68]$} & $3.91 * * *$ & 30 & 0.70 \\
\hline P DBDhi & 1.46 & 0.77 & 31 & & 1.24 & 0.61 & 31 & {$[-0.02,0.45]$} & $1.90^{\dagger}$ & 30 & 0.34 \\
\hline T DBDin & 1.63 & 0.74 & 31 & & 1.21 & 0.74 & 31 & {$[0.17,0.68]$} & $3.37 * *$ & 30 & 0.61 \\
\hline T DBDhi & 1.17 & 0.69 & 31 & & 1.08 & 0.79 & 31 & {$[-0.12,0.31]$} & 0.88 & 30 & 0.16 \\
\hline P FX home & 1.09 & 0.73 & 31 & & 0.64 & 0.35 & 31 & {$[0.23,0.66]$} & $4.27 * * *$ & 30 & 0.77 \\
\hline \multirow[t]{4}{*}{ TFX school } & 1.26 & 0.62 & 31 & & 0.94 & 0.55 & 31 & {$[0.16,0.47]$} & $4.17 * * *$ & 30 & 0.75 \\
\hline & & & & ST & & & & & & & \\
\hline & Time 1 & & & & Time 2 & & & & & & \\
\hline & $M$ & $S D$ & $n$ & & $M$ & $S D$ & $n$ & $95 \% \mathrm{Cl}$ & $t$ & $d f$ & Cohen's $d$ \\
\hline P DBDin & 1.58 & 0.68 & 27 & & 1.44 & 0.72 & 27 & {$[-0.15,0.42]$} & 0.98 & 26 & 0.19 \\
\hline P DBDhi & 1.30 & 0.74 & 27 & & 1.31 & 0.71 & 27 & {$[-0.28,0.26]$} & -0.10 & 26 & 0.02 \\
\hline T DBDin & 1.62 & 0.72 & 30 & & 1.36 & 0.73 & 30 & {$[0.03,0.50]$} & $2.28^{*}$ & 29 & 0.42 \\
\hline T DBDhi & 1.25 & 0.82 & 30 & & 0.95 & 0.52 & 30 & {$[0.07,0.54]$} & $2.67 * *$ & 29 & 0.49 \\
\hline P FX home & 1.11 & 0.60 & 27 & & 0.83 & 0.52 & 27 & {$[0.03,0.52]$} & $2.31^{*}$ & 26 & 0.44 \\
\hline TFX school & 1.35 & 0.54 & 30 & & 1.03 & 0.58 & 30 & {$[0.10,0.54]$} & $2.99 * *$ & 29 & 0.55 \\
\hline
\end{tabular}

Note. $\mathrm{CAT}=$ culturally adapted evidence-based treatment; $\mathrm{Cl}=$ confidence interval; $\mathrm{ST}$ = standard evidencebased treatment; $\mathrm{P} / \mathrm{T}$ DBDin = parent-/teacher-reported DBD Rating Scale inattention mean; $\mathrm{P} / \mathrm{T} \mathrm{DBDhi}=$ parent-/teacher-reported DBD Rating Scale hyperactivity/impulsivity mean; P FX home = parent-reported ADHDFX Scale home impairment mean; T FX school = teacher-reported ADHD-FX Scale school impairment mean. $+p \leq .10 .{ }^{*} p \leq .05 . * * p \leq .01 .{ }^{* * *} p \leq .001$. 
Finally, repeated-measures ANOVAs were employed to examine whether CAT resulted in greater improvements in ADHD symptomatology and functioning than standard EBT. Specifically, pre-post change was the withinsubjects factor, treatment condition was the between-subjects factor, and Time $\times$ Treatment Condition was the interaction term. Given that the focus of these ANOVAs was to examine significant interaction effects, only those findings are reported. One marginally significant interaction emerged for parent-reported inattention, $F(1$, $56)=3.03, p<.10$. As can be seen in Figure 2, parents who participated in CAT reported greater improvement in inattention following treatment than parents who participated in standard EBT. The effect size was medium. No other Time $\times$ Treatment Condition interactions emerged.

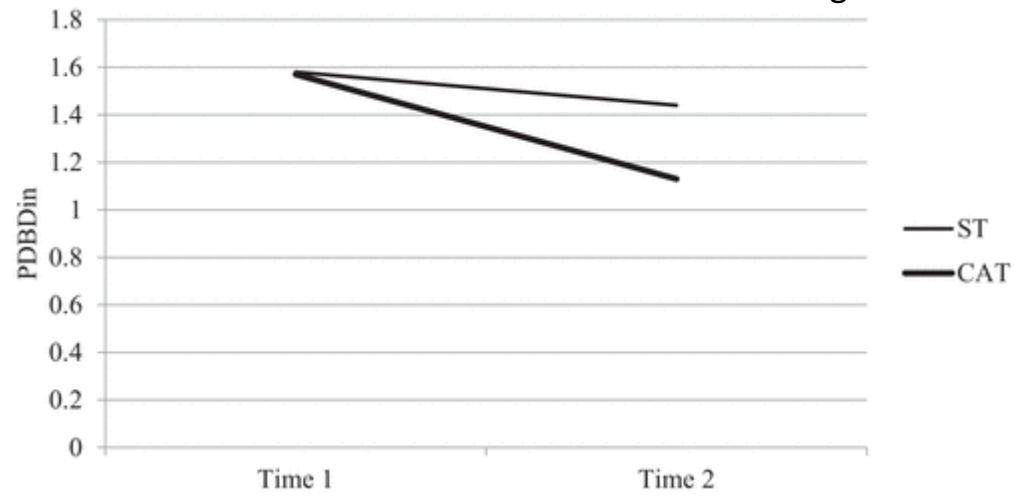

Figure 2. Change in parent-reported inattention from pretreatment (Time 1) to posttreatment (Time 2) by treatment condition.

Note. DBDin = parent-reported DBD Rating Scale inattention mean; ST = standard evidence-based treatment; CAT = culturally adapted evidence-based treatment.

Aim 3-Parental and family functioning outcomes

Paired-samples $t$ test were employed to examine whether CAT resulted in improvements in parental functioning. Specifically, $t$ tests compared pre-post treatment scores on six outcomes-mother- and fatherreported parenting stress, parental efficacy, and chaos in the home. As Table 4 indicates, significant findings emerged for mother- and father-reported parenting stress and parental efficacy, as well as mother-reported chaos. Examination of the means demonstrated that following CAT, mothers and fathers reported less parenting stress and greater parental efficacy, and mothers reported less chaos in the home. The effect sizes for all significant findings were medium to large. For comparison, paired-samples $t$ tests for standard EBT also are presented in Table 4.

Table 4. Results of Paired Samples t Tests for Parental and Family Functioning Outcomes at Pretreatment (Time 1) and Posttreatment (Time 2).

\begin{tabular}{|l|l|l|l|l|l|l|l|l|l|l|l|}
\hline & Time 1 & & & & Time 2 & & & & & \\
\hline & $\boldsymbol{M}$ & SD & $\boldsymbol{n}$ & & $\mathbf{M}$ & $\mathbf{S D}$ & $\boldsymbol{n}$ & $\mathbf{9 5 \%} \mathbf{C l}$ & $\boldsymbol{t}$ & $\boldsymbol{d}$ & Cohen's d \\
\hline M PSI & 85.23 & 18.17 & 30 & & 74.13 & 19.42 & 30 & {$[4.76,17.44]$} & $3.58^{* * *}$ & 29 & 0.65 \\
\hline M PSOC & 4.35 & 0.74 & 30 & & 4.91 & 0.63 & 30 & {$[-0.82,-0.30]$} & $-4.43 * * *$ & 29 & 0.81 \\
\hline M CHAOS & 43.60 & 9.77 & 30 & & 38.53 & 9.90 & 30 & {$[1.29,8.84]$} & $2.75 * *$ & 29 & 0.50 \\
\hline F PSI & 82.00 & 25.32 & 16 & & 75.69 & 20.88 & 16 & {$[0.13,12.50]$} & $2.18^{*}$ & 15 & 0.54 \\
\hline F PSOC & 4.12 & 0.93 & 16 & & 4.94 & 0.59 & 16 & {$[-1.24,-0.40]$} & $-4.17^{* * *}$ & 15 & 1.04 \\
\hline F CHAOS & 40.44 & 12.47 & 16 & & 37.63 & 9.11 & 16 & {$[-2.55,8.18]$} & 1.12 & 15 & 0.28 \\
\hline & & & & ST & & & & & & & \\
\hline
\end{tabular}




\begin{tabular}{|c|c|c|c|c|c|c|c|c|c|c|}
\hline & & Time 1 & & & Time 2 & & & & & \\
\hline & $M$ & $S D$ & $n$ & $M$ & $S D$ & $n$ & $95 \% \mathrm{Cl}$ & $t$ & $d f$ & Cohen's d \\
\hline M PSI & 87.37 & 21.42 & 27 & 80.15 & 23.92 & 27 & {$[0.05,14.40]$} & $2.07^{*}$ & 26 & 0.40 \\
\hline M PSOC & 4.04 & 0.86 & 27 & 4.69 & 0.73 & 27 & {$[-0.98,-0.34]$} & $-4.23 * * *$ & 26 & 0.81 \\
\hline M CHAOS & 42.26 & 9.27 & 27 & 41.63 & 11.60 & 27 & {$[-2.79,4.05]$} & 0.38 & 26 & 0.07 \\
\hline F PSI & 85.22 & 13.50 & 9 & 83.44 & 11.33 & 9 & {$[-5.12,8.67]$} & 0.60 & 8 & 0.20 \\
\hline F PSOC & 4.17 & 0.82 & 9 & 5.10 & 0.34 & 9 & {$[-1.69,-0.15]$} & $-2.76^{*}$ & 8 & 0.92 \\
\hline F CHAOS & 43.00 & 6.54 & 9 & 36.89 & 9.51 & 9 & {$[-0.90,13.12]$} & $2.01^{\dagger}$ & 8 & 0.67 \\
\hline
\end{tabular}

Finally, using the same approach as previously described, repeated-measures ANOVAs were employed to examine whether CAT resulted in greater improvements in parental functioning than standard EBT. One marginally significant interaction emerged for mother-reported chaos, $F(1,56)=2.9, p<.10$. As can be seen in Figure 3, mothers who participated in CAT reported greater improvement in chaos in the home following treatment than parents who participated in standard EBT. The effect size was medium. No other Time $x$ Treatment Condition interactions emerged.

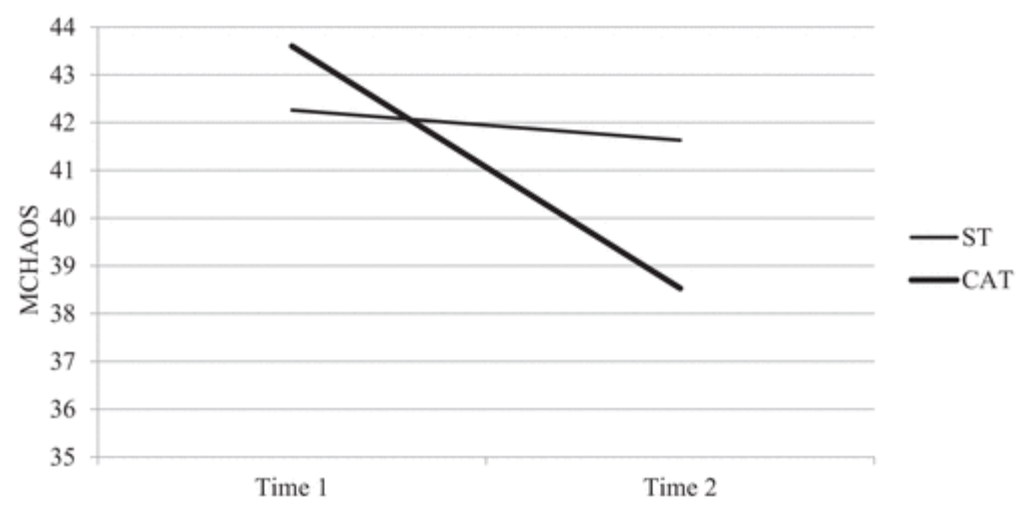

Figure 3. Change in mother-reported chaos from pretreatment (Time 1) to posttreatment (Time 2) by treatment condition.

Note. CHAOS = Chaos, Hubbub, and Order Scale sum; ST = standard evidence-based treatment; CAT = culturally adapted evidence-based treatment.

\section{Discussion}

To combat current mental health disparities in the United States, research is needed on effective treatments for many childhood disorders in ethnic minority youth. This need is particularly strong for ADHD given its prevalence and developmental trajectory, and for Latino youth who are estimated to account for nearly $40 \%$ of the children in the United States by 2060 (Alegría et al., 2010; American Psychiatric Association, 2013; Eiraldi \& Diaz, 2010; U.S. Census Bureau, 2015). Providing effective treatment for ADHD in Latino youth will likely prevent continued difficulties and impairment in adulthood, which will lessen future mental health disparities for Latino adults. Thus, the current feasibility and pilot study was the first to compare CAT for ADHD to standard EBT, and it was the first to examine treatment outcomes for ADHD in Latino youth from families less oriented to U.S. mainstream culture. Apart from two trends favoring CAT, CAT and standard EBT were equally effective when examining traditional treatment outcomes. Both resulted in significant improvements in parent- and teacherreported ADHD symptoms and functional impairment, as well as mother- and father-reported parental functioning. This is impressive considering the robustness of standard EBT, especially when delivered by culturally competent staff. CAT outperformed standard EBT when examining several engagement and acceptability outcomes. 


\section{Engagement and Acceptability Outcomes}

One of the primary aims of the current study was to explore whether CAT results in better engagement and acceptability outcomes (i.e., parental attendance, retention, engagement, and satisfaction) than standard EBT. This was an integral aim, as previous research indicates that ethnic minority families tend to be less engaged in psychosocial treatments and are more likely to drop out of treatment than European American families (Flores \& The Committee on Pediatric Research, 2010; Nock \& Ferriter, 2005), which puts them at an obvious disadvantage to benefit from treatment. Families who participated in CAT demonstrated greater engagement in treatment by completing their homework more frequently than families who participated in standard EBT, and mothers reported greater satisfaction with CAT than standard EBT. Similarly, trends demonstrated that parents who participated in CAT attended more treatment sessions/classes and were more likely to complete treatment than parents who participated in standard EBT. This is particularly impressive given that both treatment conditions experienced high rates of parental attendance, engagement, retention, and satisfaction with treatment. In addition, it is noteworthy that more fathers participated in CAT than in standard EBT. More research is needed to identify which adaptations in particular may have been helpful in promoting father participation in treatment.

The current findings support previous arguments that culturally adapted interventions may be necessary to increase both retention and engagement of ethnic minority families in psychosocial treatments (Kumpfer, Alvarado, Smith, \& Bellamy, 2002). Furthermore, given that parental attendance, engagement, and retention are integral components to families benefiting from treatment and observing improvements in more traditional treatment outcomes, the current study provides additional support for the growing body of literature highlighting the need for and benefits of culturally adapted treatments for ethnic minority families (Griner \& Smith, 2006; Huey \& Polo, 2008; Miranda et al., 2005).

It is important to note that CAT included several adaptations that were not solely related to culture (e.g., location and home visits). CAT was conducted at a neighborhood community center serving Latino families, whereas standard EBT was held at a university-based clinic. CAT also included two home visits that were not part of standard EBT. These setting and dosage differences may fully or partially explain why CAT resulted in greater parental engagement and satisfaction with treatment than standard EBT. As Baumann and colleagues (2015) point out in their review, cultural adaptation research still needs to tease apart whichadaptations are essential to treatment efficacy.

\section{ADHD Symptomatology and Functioning Outcomes}

The other two aims of the current study involved examination of more traditional treatment outcomes. Specifically, the second aim was to explore whether CAT results in improvements in ADHD symptomatology and functioning and to explore whether CAT results in similar or greater improvements than standard EBT. As expected, parents and teachers reported less inattention and less functional impairment at the end of CAT relative to baseline. Similarly, a trend also indicated that parents reported less hyperactivity/impulsivity following CAT relative to baseline. Importantly, these findings are even more robust than those reported for the initial pilot of CAT ( $n=5$; Gerdes et al., 2015).

With one exception for parent-reported inattention, CAT did not statistically differ from standard EBT when examining ADHD symptomatology and functioning outcomes. Similar to CAT, standard treatment resulted in improvements in most outcome measures, including teacher-reported ADHD symptoms and parent- and teacher-reported functioning. This is most likely due to the strength of both interventions that were delivered by culturally competent staff. As has been noted in existing studies (e.g., McCabe \& Yeh, 2009), it is very difficult to statistically outperform an established, evidence-based intervention. In the current study, standard EBT also was delivered by culturally competent staff, the importance of which cannot be understated and likely resulted in an improved EBT even without any adaptations specific to culture. For example, coleaders were attuned to cultural 
beliefs that may result in parental discomfort about some sessions (e.g., time out) and were open to discussing and validating parental concerns as part of the treatment session.

These are important findings, as they are the first published findings demonstrating the effectiveness of both standard PMT, as well as culturally adapted PMT at improving child behavior in Latino youth with ADHD from families less oriented to U.S. mainstream culture. Until now, research examining PMT had established its effectiveness at improving child behavior in youth with ADHD from predominantly European American families or Latino families more oriented to U.S. mainstream culture (Arnold et al., 2003; Pelham \& Fabiano, 2008).

\section{Parental and Family Functioning Outcomes}

The final aim was to explore whether CAT results in improvements in parental and family functioning (i.e., parenting stress, parental efficacy, and family chaos) and to explore whether CAT results in similar or greater improvements than standard EBT. As expected, mothers and fathers reported improvements in parenting stress and parental efficacy and mothers reported less chaos in the home following CAT relative to baseline. Similar to the previously reported child outcomes, these findings are even stronger than expected based on the initial, small pilot of CAT (Gerdes et al., 2015).

With one exception for mother-reported chaos, CAT did not statistically differ from standard EBT when examining parental functioning outcomes. Standard EBT also resulted in improvements in maternal parenting stress, as well as maternal and paternal parental efficacy. As mentioned previously, the robustness of both interventions likely made it difficult for CAT to statistically outperform standard EBT, especially when delivered by culturally competent staff.

Previous research has documented that relative to parents of comparison youth, parents of youth with ADHD experience poorer functioning in many domains, including their own mental health, as well as their cognitions and affect related to parenting (for a review, see Johnston \& Mash, 2001). Thus, the current findings are particularly noteworthy, as they add to the small body of ADHD treatment outcome literature demonstrating improvements in parental functioning following PMT for youth with ADHD (Gerdes et al., 2012). Improvements in parental functioning likely are crucial to ensuring that parents continue to consistently implement the skills and strategies taught during PMT, which will allow for the maintenance of child improvements.

\section{Limitations}

Despite the important contributions of the current feasibility and pilot study, several limitations are worth noting. Due to the pilot nature of the study, the number of families per treatment condition was relatively small (approximately 30), and the number of fathers per condition was even smaller (approximately 10-15), despite holding groups in the evening and providing childcare. Effective methods to increase father involvement are needed, as it is possible that father participation in treatment may result in improved outcomes for the family (Lundahl, Tollefson, Risser, \& Lovejoy, 2007). The lack of follow-up data also is a limitation, as we are unable to establish whether treatment gains were sustained over time. Given both geographic parameters and primary aims of the study, the current sample also included a relatively homogeneous sample of Latino families; most were of Mexican decent, most were less oriented to U.S. mainstream culture and more oriented to traditional Latino culture, and most fell on the lower end of SES. Although this was the intended sample for the initial pilot study, a larger, more diverse sample of Latino families would provide more power to detect statistically significant differences, as well as the ability to examine potential moderators of treatment outcomes, such as Latino subgroup, parental acculturation, and SES.

\section{Clinical Implications and Future Directions}

In sum, findings from the current feasibility and pilot study are promising. This was the first study to compare a CAT for ADHD with standard EBT and the first to examine treatment outcomes for ADHD in school-aged Latino 
youth from families less oriented to U.S. mainstream culture. Both CAT and EBT resulted in significant improvements when examining traditional treatment outcomes (i.e., parent- and teacher-reported ADHD symptoms and functional impairment, as well as mother- and father-reported parental functioning). This is impressive considering the robustness of standard EBT, especially when delivered by culturally competent staff. Although identification of the exact adaptations responsible is still needed (e.g., cultural versus setting and/or dosage), CAT outperformed standard EBT when examining several engagement and acceptability outcomes.

As mentioned previously, replication of the current study with a larger, more diverse sample of Latino families is needed, including a greater representation of fathers. This would allow for examination of possible moderators (e.g., parental acculturation and SES) and provide valuable information on which families may benefit most from CAT versus standard EBT. The need to examine longitudinal, follow-up data also is warranted, as it is important to develop a better understanding of long-term outcomes for youth and their families participating in treatment. Consistent with Baumann and colleagues (2015) recommendations, another critical area for future research involves community dissemination. To ensure that as many Latino families as possible can access CAT, community partnership is needed. Thus, an important next step is to train existing community mental health professionals to deliver the treatment and to examine the effectiveness of CAT when delivered within a community setting by community providers. Finally, future research directly comparing several distinct cultural adaptations of the same treatment is needed to tease apart which adaptations are essential to treatment efficacy (Baumann et al., 2015).

\section{Declaration of Conflicting Interests}

The author(s) declared no potential conflicts of interest with respect to the research, authorship, and/or publication of this article.

\section{Funding}

The author(s) disclosed receipt of the following financial support for the research, authorship, and/or publication of this article: This research was supported by the Eunice Kennedy Shriver National Institute of Child Health \& Human Development of the National Institutes of Health under Award Number R21HD078553. The content is solely the responsibility of the authors and does not necessarily represent the official views of the National Institutes of Health.

\section{References}

Abidin, R. R. (2012). Parenting Stress Index (4th ed.). Lutz, FL: Psychological Assessment Resources.

Achenbach, T. M., \& Rescorla, L. A. (2001). Manual for the ASEBA school-age forms \& profiles. Burlington: Research Center for Children, Youth, \& Families, University of Vermont.

Alegría, M., Vallas, M., \& Pumariega, A. (2010). Racial and ethnic disparities in pediatric mental health. Child and Adolescent Psychiatric Clinics of North America, 19, 759-774.

Alegría, M., \& Woo, M. (2009). Conceptual issues in Latino mental health. In F. A. Villarruel, G. Carlo, J. M. Grau, M. Amity, N. J. Cabrera, \& T. J. Schahin (Eds.), Handbook of U.S. Latino psychology: Developmental and community-based perspectives (pp. 15-30). Thousand Oaks, CA: SAGE.

American Psychiatric Association. (2013). Diagnostic and statistical manual of mental disorders (5th ed.). Arlington, VA: American Psychiatric Publishing.

Arcia, E., Fernández, M. C., \& Jáquez, M. (2004). Latina mothers' stances on stimulant medication: Complexity, conflict, and compromise. Journal of Developmental \& Behavioral Pediatrics, 25, 311-317.

Arnold, L. E., Elliott, M., Sachs, L., Bird, H., Kraemer, H. C., Wells, K. C., . . Wigal, T. (2003). Effects of ethnicity on treatment attendance, stimulant response/dose, and 14-month outcome in ADHD. Journal of Consulting and Clinical Psychology, 71, 713-727.

Barkley, R. A. (1997). Defiant children: A clinician's manual for assessment and parent training. New York, NY: Guilford Press. 
Baumann, A. A., Powell, B. J., Kohl, P. L., Tabak, R. G., Penalba, V., Proctor, E. K., . . Cabassa, L. J. (2015). Cultural adaptation and implementation of evidence-based parent-training: A systematic review and critique of guiding evidence. Child and Youth Services Review, 53, 113-120.

Bernal, G., \& Domenech Rodríguez, M. M. (Eds.). (2012). Cultural adaptations: Tools for evidence-based practice with diverse populations. Washington, DC: American Psychological Association.

Bernal, G., Jiménez-Chafey, M. I., \& Domenech Rodríguez, M. M. (2009). Cultural adaptation of treatments: A resource for considering culture in evidence-based practice. Professional Psychology: Research and Practice, 40, 361-368.

Biederman, J., Petty, C. R., Woodworth, K. Y., Lomedico, A., Hyder, L. L., \& Faraone, S. V. (2012). Adult outcome of attention-deficit/hyperactivity disorder: A controlled 16-year follow-up study. Journal of Clinical Psychiatry, 73, 941-950.

Cabassa, L. J., Zayas, L. H., \& Hansen, M. C. (2006). Latino adults' access to mental health care: A review of epidemiological studies. Administration and Policy in Mental Health and Mental Health Services Research, 33, 316-330.

Centers for Disease Control and Prevention. (2018). ADHD, data and statistics. Retrieved from https://www.cdc.gov/ncbddd/adhd/data.html

Cuellar, I., Arnold, B., \& Maldonado, R. (1995). Acculturation Rating Scale for Mexican Americans-II: A revision of the original ARSMA scale. Hispanic Journal of Behavioral Sciences, 17, 275-304.

Davanzo, P., Kerwin, L., Nikore, V., Esparza, C., Forness, S., \& Murrelle, L. (2004). Spanish translation and reliability testing of the Child Depression Inventory. Child Psychiatry \& Human Development, 35, 75-92.

Eiraldi, R., \& Diaz, Y. (2010). Use of treatment services for attention-deficit/hyperactivity disorder in Latino children. Current Psychiatry Reports, 12, 403-408.

Eisenstadt, T. H., Eyberg, S., McNeil, C. B., Newcomb, K., \& Funderburk, B. (1993). Parent-Child Interaction Therapy with behavior problem children: Relative effectiveness of two stages and overall treatment outcome. Journal of Clinical Child Psychology, 22, 42-51.

Eyberg, S. M. (1993). Consumer satisfaction measures for assessing parent training programs. In L. VandeCreek, S. Knapp, \& T. L. Jackson (Eds.), Innovations in clinical practice: A source book (Vol. 12, pp. 377-382). Sarasota, FL: Professional Resource Press.

Flores, G., \& The Committee on Pediatric Research. (2010). Racial and ethnic disparities in the health and health care of children. Pediatrics, 125, e979-e1020. Gerdes, A. C., Haack, L. M., \& Schneider, B. W. (2012). Parental functioning in families of children with ADHD: Evidence for behavioral parent training and importance of clinical meaningfulness. Journal of Attention Disorders, 16, 147-156.

Gerdes, A. C., Kapke, T. L., Lawton, K. E., Grace, M., \& Dieguez Hurtado, G. (2015). Culturally adapting parent training for Latino youth with ADHD: Development and pilot. Journal of Latina/o Psychology, 3, 71-87.

Gerdes, A. C., Lawton, K. E., Haack, L. M., \& Dieguez Hurtado, G. (2013). Assessing ADHD in Latino families: Evidence for moving beyond symptomatology. Journal of Attention Disorders, 17, 128-140.

Griner, D., \& Smith, T. B. (2006). Culturally adapted mental health interventions: A meta-analytic review. Psychotherapy: Theory, Research, Practice, Training, 43, 531-548.

Haack, L. M., Gerdes, A. C., Lawton, K. E., \& Schneider, B. W. (2016). Understanding and measuring functional impairment in diverse children with ADHD: Development of the ADHD-FX scale with an at-risk, community sample. Journal of Attention Disorders, 20, 487-500. doi:10.1177/1087054714527791

Haack, L. M., Gerdes, A. C., Schneider, B. W., \& Dieguez Hurtado, G. (2011). Advancing our knowledge of ADHD in Latino children: Psychometric and cultural properties of Spanish-versions of parental/family functioning measures. Journal of Abnormal Child Psychology, 39, 33-43.

Haack, L. M., Gonring, K., Harris, M., Gerdes, A. C., \& Pfiffner, L. J. (2016). Assessing impairment in childhood ADHD: Validation of the parent and teacher ADHD-FX rating scale in a dual-site clinical sample. Journal of Attention Disorders. Advance online publication. doi: 10.1177/1087054716659360

Hollingshead, A. A. (1975). Four-factor index of social status. Unpublished manuscript, Yale University, New Haven, $\mathrm{CT}$. 
Huey, S. J., \& Polo, A. J., Jr. (2008). Evidence-based psychosocial treatments for ethnic minority youth. Journal of Clinical Child \& Adolescent Psychology, 37, 262-301.

Johnston, C., \& Mash, E. J. (1989). A measure of parenting satisfaction and efficacy. Journal of Clinical Child Psychology, 18, 167-175.

Johnston, C., \& Mash, E. J. (2001). Families of children with attention-deficit/hyperactivity disorder: Review and recommendations for future research. Clinical Child and Family Psychology Review, 4, 183-207.

Kim, R. E., Lau, A. S., \& Chorpita, B. F. (2016). The impact of Latino caregiver acculturation on treatment engagement in children's community mental health services. Journal of Child and Family Studies, 25, 891-901.

Knight, G. P., Gonzales, N. A., Saenz, D. S., Bonds, D. D., German, M., ... Updegraff, K. A. (2010). The Mexican American Cultural Values Scale for adolescents and adults. Journal of Early Adolescence, 30, 444-481.

Kouyoumdjian, H., Zamboanga, B. L., \& Hansen, D. J. (2003). Barriers to community mental health services for Latinos: Treatment considerations. Clinical Psychology: Science and Practice, 10, 394-422.

Kovacs, M., \& MHS Staff. (2011). The Children's Depression Inventory 2 Manual. North Tonawanda, NY: MultiHealth Systems.

Kumpfer, K. L., Alvarado, R., Smith, P., \& Bellamy, N. (2002). Cultural sensitivity and adaptation in family-based prevention interventions. Prevention Science, 3, 241-246.

Lahey, B. B., Pelham, W. E., Loney, J., Kipp, H., Ehrhardt, A., Lee, S. S., . . Massetti, G. (2004). Three-year predictive validity of DSM-IV attention deficit hyperactivity disorder in children diagnosed at 4-6 years of age. American Journal of Psychiatry, 161, 2014-2020.

Leibach, G. G., Perrin, P. B., Nicholls, E., Olivera, S. L., Quintero, L. M., Trujillo, D. M. V., \& Arango-Lasprilla, J. C. (2015). Health related quality of life and mental health in children with SCI/D from Neiva, Colombia. NeuroRehabilitation, 36, 215-221.

Leslie, L. K., Lambros, K. M., Aarons, G. A., Haine, R. A., \& Hough, R. L. (2008). School-based service use by youth with ADHD in public-sector settings. Journal of Emotional and Behavioral Disorders, 16, 163-177.

Lundahl, B. W., Tollefson, D., Risser, H., \& Lovejoy, M. C. (2007). A meta-analysis of father involvement in parent training. Research on Social Work Practice, 18, 97-106.

Matheny, A. P., Wachs, T. D., Ludwig, J. L., \& Phillips, K. (1995). Bringing order out of chaos: Psychometric characteristics of the Confusion, Hubbub, and Order Scale. Journal of Applied Developmental Psychology, 16, 429-444.

McCabe, K., \& Yeh, M. (2009). Parent-Child Interaction Therapy for Mexican Americans: A randomized clinical trial. Journal of Clinical Child \& Adolescent Psychology, 38, 753-759.

McCabe, K., Yeh, M., Lau, A., \& Argote, C. B. (2012). Parent child interaction therapy for Mexican Americans: Results of a pilot randomized clinical trial at follow-up. Behavior Therapy, 43, 606-618.

Miranda, J., Bernal, G., Lau, A., Kohn, L., Hwang, W., \& LaFromboise, T. (2005). State of the science on psychosocial interventions for ethnic minorities. Annual Review of Clinical Psychology, 1, 113-142.

Morgan, P. L., Hillemeier, M. M., Farkas, G., \& Maczuga, S. (2014). Racial/ethnic disparities in ADHD diagnosis by kindergarten entry. Journal of Child Psychology and Psychiatry, 55, 905-913.

Nock, M. K., \& Ferriter, C. (2005). Parent management of attendance and adherence in child and adolescent therapy: A conceptual and empirical review. Clinical Child and Family Psychology Review, 8, 149-166.

Pelham, W. E. (2002). Attention deficit hyperactivity disorder: Diagnosis, assessment, nature, etiology, and treatment. Unpublished manuscript, State University of New York at Buffalo.

Pelham, W. E., \& Fabiano, G. A. (2008). Evidence-based psychosocial treatments for attentiondeficit/hyperactivity disorder. Journal of Child \& Adolescent Psychology, 37, 184-217.

Pelham, W. E., Gnagy, E. M., Greenslade, K. E., \& Milich, R. (1992). Teacher ratings of DSM-III-R symptoms for the disruptive behavior disorders. Journal of the American Academy of Child \& Adolescent Psychiatry, 31, 210-218.

Reynolds, C. R., \& Richmond, B. O. (2008). Revised Children's Manifest Anxiety Scale-Second Edition manual. Los Angeles, CA: Western Psychological Services. Rubio-Stipec, M., Bird, H., Canino, G., \& Gould, M. (1990). 
The internal consistency and concurrent validity of a Spanish translation of the child behavior checklist. Journal of Abnormal Child Psychology, 18, 393-406.

Steidel, A. G. L., \& Contreras, J. M. (2003). A new familism scale for use with Latino populations. Hispanic Journal of Behavioral Sciences, 25, 312-330.

Sue, S., Zane, N., Nagayama Hall, G. C., \& Berger, L. K. (2009). The case for cultural competency in psychotherapeutic interventions. Annual Review of Psychology, 60, 525-548.

U.S. Census Bureau. (2015). Projections of the size and composition of the U.S. population: 2014-2060. Retrieved from https://www.census.gov/library/publications/2015/demo/p25-1143.html

Visser, S. N., Bitsko, R. H., Danielson, M. L., Perou, R., \& Blumberg, S. J. (2010). Increasing prevalence of parentreported attentiondeficit/hyperactivity disorder among children-United States, 2003 and 2007. Morbidity and Mortality Weekly Report, 59, 1439-1443. 